\title{
REALISTIC SIMULATION OF OCEAN SURFACE USING WAVE SPECTRA
}

\author{
Jocelyn Fréchot \\ LaBRI - Laboratoire bordelais de recherche en informatique \\ Domaine universitaire, 351 cours de la Libération, 33405 Talence CEDEX, France \\ jocelyn.frechot@labri.fr
}

Keywords: Natural phenomena, realistic ocean waves, procedural animation, parametric energy spectra

\begin{abstract}
We present a method to simulate ocean surfaces away from the coast, with correct statistical wave height and direction distributions. By using classical oceanographic parametric wave spectra, our results fit real world measurements, without depending on them. Since wave spectra are independent of the ocean model, Gerstner parametric equations and Fourier transform method can be used with them. Moreover, since they are simple to use and need very few parameters, they allow easy production of ocean surface animations usable in movies and games. We explain how to accurately sample them, to achieve oceanic waves in deep water according to given wind parameters, in a realistic way.
\end{abstract}

\section{INTRODUCTION}

The reproductions of many natural phenomena is still an open problem in Computer Graphics. In particular, the realistic simulation of oceanic surfaces continues to be of great interest. Many fields of activity rely on it: virtual reality, movies and games, but also sailing (Cieutat et al., 2001) and oceanographic simulations, among others.

While beautiful pictures and animations such as wave refraction (Gonzato and le Saëc, 2000) (Gamito and Musgrave, 2002) are produced, there is still a need for oceanic scenes with realistic wave features. Models based on fluid mechanics equations (Enright et al., 2002) (Mihalef et al., 2004) should give the more realistic results, but are inadequate for large oceanic surfaces.

For twenty years, procedural models for wave representation in Computer Graphics have been developed and improved (Iglesias, 2004). The Gerstner parametric equations and their Fourier transform rewriting are well known by the oceanographic community. Based on the linear wave theory, they allow representation of natural wave shapes and moves in deep water above the capillary size. As they not describe any net mass transport, they are limited to nonbreaking waves and to scenes without violent storms.

Wave height measurement data allow to simulate a given sea state. In the more general case, oceanic sur- face statistics are taken in account by the use of parametric wave spectra. For given wind parameters, they indicate the distribution of wave energy as a function of frequency. However, none of the existing methods are able to correctly reproduce sea states described by these spectra.

In this paper, we explain how wave spectra are related to oceanic surface energy and we detail a method to deduce wave heights from it. We propose a solution for optimal spectrum sampling, suitable for parametric and Fourier transform models. This method enables the accurate simulation of oceanic surface. Users can easily produce realistic wave animations, with respect to supplied weather conditions.

The structure of this paper is as follows. Section 2 summarizes ocean models for Computer Graphics. In section 3, we detail our method and give classical parametric spectrum examples. We handle wave statistics in section 4 . We discuss our results in section 5 , and conclude in section 6 .

\section{OCEAN MODELS}

\subsection{Definitions and relationships}

The surface elevation, $\eta$, is the vertical displacement from the mean water level of the oceanic surface at 
given point and time. The wave amplitude, $a$, is the maximum surface elevation due to this wave. The wave height, $H$, is the vertical distance between a trough and an adjacent crest. For a single wave, $H=2 a$. The wavelength, $\lambda$, is the distance between two successive crests. The period, $T$, is the time interval between the passage of successive crests through a fixed point. The frequency, $f=1 / T$, is the number of crests that pass a fixed point in 1 second. The wave speed or phase speed, $c=\lambda / T$, is the speed of wave crests or troughs. It can also be expressed as $c=\omega / \kappa$, where $\omega=2 \pi f$ is the angular velocity or angular frequency, and $\kappa=2 \pi / \lambda$ is the wavenumber. The water depth, $d$, is the vertical distance between the floor and the mean water level.

The concept of deep water is wave dependent and is related to the ratio of the water depth to the wavelength of the wave. This comes from the transcendental expression

$$
\lambda=\frac{g T^{2}}{2 \pi} \tanh \left(\frac{2 \pi d}{\lambda}\right),
$$

where $g \approx 9.807 \mathrm{~m} \cdot \mathrm{s}^{-2}$ is the standard acceleration of gravity. Since $\tanh (x) \approx 1$ when $x>\pi$, and $\tanh (x) \approx x$ when $x<\pi / 10$, water is considered deep if $d / \lambda>1 / 2$ and shallow if $d / \lambda>1 / 20$. This leads to some simplifications in the expressions of the terms given above (see table 1).

Table 1: Relations between oceanographic terms. The subscript $\infty$ indicates a deep water term.

$$
\begin{array}{l|l}
\text { Transitional depth: } & \begin{array}{l}
\text { Deep water: } \\
1 / 20<d / \lambda<1 / 2
\end{array} \\
\hline c=\frac{\lambda}{T}=\frac{\omega}{\kappa} & \\
c=\frac{g T}{2 \pi} \tanh (\kappa d) & c_{\infty}=\frac{g T}{2 \pi} \\
c^{2}=\frac{g}{\kappa} \tanh (\kappa d) & c_{\infty}^{2}=\frac{g}{\kappa}=\left(\frac{g}{\omega}\right)^{2} \\
\kappa=\frac{2 \pi}{\lambda} & \\
\kappa_{\infty}=\kappa \tanh (\kappa d) & \\
\lambda=\frac{2 \pi}{\kappa}=c T & \\
\lambda=\frac{g T^{2}}{2 \pi} \tanh \left(\frac{2 \pi d}{\lambda}\right) & \\
\lambda=\lambda=\lambda \tanh (\kappa d) & \\
\omega=\frac{2 \pi}{T}=\kappa c & \\
\omega^{2}=g \kappa \tanh (\kappa d)=g \kappa_{\infty} & \omega_{\infty}^{2}=g \kappa
\end{array}
$$

\subsection{Parametric equations}

The base model for ocean waves simulation comes from the linear (or small-amplitude) wave theory by
(Airy, 1845), widely used in oceanic engineering, and in Computer Graphics by (Peachey, 1986). It describes waves with a sinusoidal shape, which corresponds to calm weather conditions. Considering a location $x_{0}$ lying on a $1 \mathrm{D}$ surface at rest, the elevation $z=\eta$ at time $t$ due to a wave with wavenumber $\kappa$, amplitude $a$ and angular velocity $\omega=\sqrt{g \kappa}$ is

$$
z\left(x_{0}, t\right)=a \cos \left(\kappa x_{0}-\omega t\right) .
$$

When the steepness of the wave increases, its crests become sharper and its troughs flatter. Therefore, (Fournier and Reeves, 1986) used a more realistic description based on trochoids, made by (von Gerstner, 1804) and by (Rankine, 1863). The surface equation is now

$$
\left\{\begin{array}{l}
x\left(x_{0}, t\right)=x_{0}+a \sin \left(\kappa x_{0}-\omega t\right) \\
z\left(x_{0}, t\right)=z_{0}-a \cos \left(\kappa x_{0}-\omega t\right),
\end{array}\right.
$$

where $z_{0}$ is the surface elevation at rest (typically 0 ). Note that the important point here is the phase term difference of $-\pi / 2$ between $x\left(x_{0}, t\right)$ and $z\left(x_{0}, t\right)$, not the use of a particular sine or cosine function. This Lagrangian model describes the trajectory in a vertical plane of a particle $(x, z)$ around its position at rest $\left(x_{0}, z_{0}\right)$. Summing several waves and extending equation 3 to a $2 \mathrm{D}$ surface gives

$$
\left\{\begin{array}{l}
\vec{x}\left(\vec{x}_{0}, t\right)= \\
\vec{x}_{0}+\sum_{\vec{\kappa}} \hat{\vec{\kappa}} a(\vec{\kappa}) \sin \left(\vec{\kappa} \cdot \vec{x}_{0}-\omega(\kappa) t+\phi(\vec{\kappa})\right) \\
z\left(\vec{x}_{0}, t\right)= \\
z_{0}-\sum_{\vec{\kappa}} a(\vec{\kappa}) \cos \left(\vec{\kappa} \cdot \vec{x}_{0}-\omega(\kappa) t+\phi(\vec{\kappa})\right),
\end{array}\right.
$$

where $\vec{x}=(x, y)$ is the horizontal particle position at time $t, \vec{x}_{0}=\left(x_{0}, y_{0}\right)$ its position at rest, $\vec{\kappa}=\left(\kappa_{x}, \kappa_{y}\right)$ a wave vector, i.e. a vector with magnitude $\|\vec{\kappa}\|=$ $\kappa$ and direction of the considered wave propagation and $\hat{\vec{\kappa}}=\vec{\kappa} /\|\vec{\kappa}\|$ is the unit vector of $\vec{\kappa}$. Because of the presence of uniformly distributed random phase terms, $\phi(\vec{\kappa}) \in[0,2 \pi[$, this model is also known as random waves. Any set of $\vec{x}_{0}$ can be taken, which allows the use of adaptive mesh, for optimal surface sampling (Hinsinger et al., 2002).

\subsection{Inverse Fourier transform}

Another method for computing equation 4 is the $2 \mathrm{D}$ inverse discrete Fourier transform, used by (Mastin et al., 1987), (Premože and Ashikhmin, 2001) and (Tessendorf, 2001). The set of $N \times M$ complex numbers $F_{n, m}$ is transformed into a set of complex numbers $f_{p, q}$ by

$$
f_{p, q}=\sum_{n=1}^{N} \sum_{m=1}^{M} F_{n, m} \exp \left(\mathrm{i} 2 \pi\left(\frac{n p}{N}+\frac{m q}{M}\right)\right),
$$


where $p \in\{1, . ., N\}$ and $q \in\{1, . ., M\}$. This is of particular interest because the fast Fourier transform (FFT) algorithm is an efficient way to compute these sum. However, in comparison with the previous method, its usage is more restrictive. The set of $\vec{x}_{0}$ is defined as a regular grid of $N \times M$ points with dimensions $L_{x} \times L_{y}$ and origin at the center, such that $\vec{x}_{0}=$ $\left(n_{x_{0}} L_{x} / N, m_{y_{0}} L_{y} / M\right)$, where $n_{x_{0}} \in[-N / 2, N / 2[$ and $m_{x_{0}} \in[-M / 2, M / 2[$ are integers. We rewrite equation 4 as

$$
\left\{\begin{array}{l}
\vec{x}\left(\vec{x}_{0}, t\right)=\vec{x}_{0}+\Im\left(\sum_{\vec{\kappa}} \hat{\vec{\kappa}} A(\vec{\kappa}, t) \exp \left(\mathrm{i} \vec{\kappa} \cdot \vec{x}_{0}\right)\right) \\
z\left(\vec{x}_{0}, t\right)=z_{0}-\Re\left(\sum_{\vec{\kappa}} A(\vec{\kappa}, t) \exp \left(\mathrm{i} \vec{\kappa} \cdot \vec{x}_{0}\right)\right),
\end{array}\right.
$$

where $\Im()$ and $\Re()$ correspond, respectively, to the imaginary part and real part of the expression, and

$$
A(\vec{\kappa}, t)=a(\vec{\kappa}) \exp (\mathrm{i}(-\omega(\kappa) t+\phi(\vec{\kappa}))) .
$$

Rather than the random phase term $\phi$, authors commonly use a normally distributed random amplitude (see section 4). To comply with equation 5 , the set of wave vectors must be

$$
\vec{\kappa}=\left(2 \pi \frac{n_{\kappa}}{L_{x}}, 2 \pi \frac{m_{\kappa}}{L_{y}}\right),
$$

where $n_{\kappa}$ and $m_{\kappa}$ are defined as $n_{x_{0}}$ and $m_{x_{0}}$ above. The resulting surface is periodic in all directions and can be used as a tile (Tessendorf, 2001).

To decrease the FFT computation time by a factor two, real numbers can be used instead of complex ones. This is achieved by adding two waves with same amplitude and travelling in opposite directions (Tessendorf, 2001). Equation 7 becomes

$$
\begin{aligned}
A(\vec{\kappa}, t)= & a(\vec{\kappa}) \exp (\mathrm{i}(-\omega(\kappa) t+\phi(\vec{\kappa}))) \\
& +a(\vec{\kappa}) \exp (-\mathrm{i}(-\omega(\kappa) t+\phi(\vec{\kappa}))) \\
= & 2 a(\vec{\kappa}) \cos (-\omega(\kappa) t+\phi(\vec{\kappa})) .
\end{aligned}
$$

This results in a single stationary wave with time dependent amplitude. This phenomenon is known as standing wave. The drawback of this method is that the generated surface is only half the size of the expected one. The second half is obtained with a rotation of the first one by 180 degrees around the origin, leading to pattern duplications.

\section{WAVE SPECTRA}

To be able to use the models discussed so far, we need a function $a(\vec{\kappa})$. For the parametric model, we also need to know which set of wave vectors characterizes the surface we want to generate. The more natural method is to use records of surface elevation measurement data used by oceanic engineering (Thon and Ghazanfarpour, 2002). Measurements are made with accelerometers mounted on buoys, satellite altimeters, high frequency radars and others techniques. A spectral analysis of the collected data is done using the Fourier transform from time domain to frequency domain. This decomposition of the surface elevation gives a histogram of wave frequencies $\omega$, with corresponding amplitudes and possibly directions, known as frequency amplitude spectrum. The same analysis method can be used with oceanic surface photographs (Tessendorf, 2001) (Thon and Ghazanfarpour, 2002).

An amplitude spectrum can be used to generate a surface with the same characteristics as the related one. However, to get several oceanic surfaces with different sea states, as many spectra are needed. Instead, we rely on parametric wave spectra, which can fit any environment conditions, like wind speed and fetch.

\subsection{Wave energy and amplitudes}

Oceanographers are mostly concerned by wave energy rather than amplitude. For this purpose, they use another type of spectrum, $S(\omega)$, called wave frequency energy spectrum or wave spectrum, which gives information about wave energy distribution as a continuous function of frequency. This is a smoothed version of a modified amplitude spectrum (see below). Directional informations are taken into account with a directional wave spectrum:

$$
E(\omega, \theta)=S(\omega) D(\omega, \theta)
$$

where $\theta$ is the direction of the wave and $D(\omega, \theta)$ is a directional spreading function, defined such that

$$
\int_{-\pi}^{+\pi} E(\omega, \theta) \mathrm{d} \theta=S(\omega)
$$

for all values of $\omega$. The Pierson-Moskowitz parametric wave spectrum (see section 3.2) has been regularly used in the field of Computer Graphics since (Mastin et al., 1987) as a direct evaluation of wave amplitudes. But this method cannot reflect statistical characteristics of the oceanic surface. First, this is a continuous function, so it needs to be integrated to be used with a discrete model. Second, wave spectrum does not give direct information about wave amplitude but energy.

The mean energy per unit surface area is $E=$ $\rho g \operatorname{var}(\eta)$, where $\rho$ is the water density and $\operatorname{var}(\eta)$ the variance of the surface elevation. The wave spectrum is defined to be related to the surface energy by

$$
\operatorname{var}(\eta)=\int_{0}^{+\infty} S(\omega) \mathrm{d} \omega .
$$


For a zero mean process, like the sinusoidal wave model from equation 2 , the variance is

$$
\operatorname{var}(x)=x_{r m s}^{2},
$$

where $x_{r m s}$ is the root mean square of the values. For a general process, this becomes $x_{r m s}^{2}-\bar{x}^{2}$. The mean amplitude of the trochoidal wave model from equation 3 is $\bar{a}=-\left(\kappa a^{2}\right) / 2$, which can be reasonably neglected in most cases for practical purposes. So, we apply equation 13 to this model as well for simplicity. The rms of a sinusoidal or trochoidal wave with amplitude $a$ is

$$
a_{r m s}^{2}=\frac{a^{2}}{2} .
$$

As we see the oceanic surface as the sum of many waves with independent random phases, the total variance of the surface elevation is the sum of the variance of each wave. So, taking a finite range of frequencies $\left[\omega_{\min }, \omega_{\max }\right]$ such that it is representative of the whole energy, i.e.

$$
\int_{\omega_{\min }}^{\omega_{\max }} S(\omega) \mathrm{d} \omega \approx \int_{0}^{+\infty} S(\omega) \mathrm{d} \omega
$$

we divide it in $N$ samples of width $\Delta \omega_{n}$ and tag the mean frequency of each of them as $\omega_{n}$ to get the relation

$$
\operatorname{var}(\eta)=\sum_{n=1}^{N} \operatorname{var}\left(a\left(\omega_{n}\right)\right)=\sum_{n=1}^{N} \frac{a\left(\omega_{n}\right)^{2}}{2} .
$$

Regarding equations 12 and 15 , this is rewritten as

$$
\operatorname{var}(\eta)=\int_{\omega_{\min }}^{\omega_{\max }} S(\omega) \mathrm{d} \omega=\sum_{n=1}^{N} \int_{\Delta \omega_{n}} S(\omega) \mathrm{d} \omega,
$$

and so we found the amplitude of each wave with

$$
a\left(\omega_{n}\right)^{2}=2 \int_{\Delta \omega_{n}} S(\omega) \mathrm{d} \omega \approx 2 S\left(\omega_{n}\right) \Delta \omega_{n} .
$$

To take into account the direction of the wave, we sample the whole directional spectrum $E(\omega, \theta)$ :

$$
a\left(\omega_{n}, \theta_{n}\right)^{2}=2 \int_{\Delta \omega_{n}} \int_{\Delta \theta_{n}} E(\omega, \theta) \mathrm{d} \theta \mathrm{d} \omega .
$$

So, for each wave with angular frequency $\omega$ and direction $\theta$, we can now compute the corresponding amplitude $a(\omega, \theta)$.

\subsection{Parametric spectra}

Parametric spectrum models are empirical expressions with user defined parameters that have been found to fit ocean surface elevation measurements. Most common parameters include wind speed and direction and fetch. They are useful to simulate realistic ocean waves without measurement data, but can also be altered to fit specific data.

The base of modern parametric wave spectrum was found by (Phillips, 1957) and has the form

$$
S_{P}(\omega)=\frac{\alpha g^{2}}{\omega^{5}}
$$

where $\alpha=0.0081$ is called the Phillips constant. Taking into account wind speed, (Pierson and Moskowitz, 1964) found an expression that describes a theoretical fully developed sea with infinite fetch (see figure 1). The wind has blown with no change over a large area for several hours, and waves growth is almost null. The spectrum is

$$
S_{P M}(\omega)=S_{P}(\omega) \exp \left(-\frac{5}{4}\left(\frac{\omega_{p}}{\omega}\right)^{4}\right),
$$

where $\omega_{p}$ is the frequency of the peak of the spectrum, i.e. $\mathrm{d} S_{P M} / \mathrm{d} \omega=0$ for $\omega_{p}$. Usual value for $\omega_{p}$ is

$$
\omega_{p}=\frac{0.855 g}{U_{10}},
$$

where $U_{10}$ is the wind speed at a height of 10 meters above the sea surface.

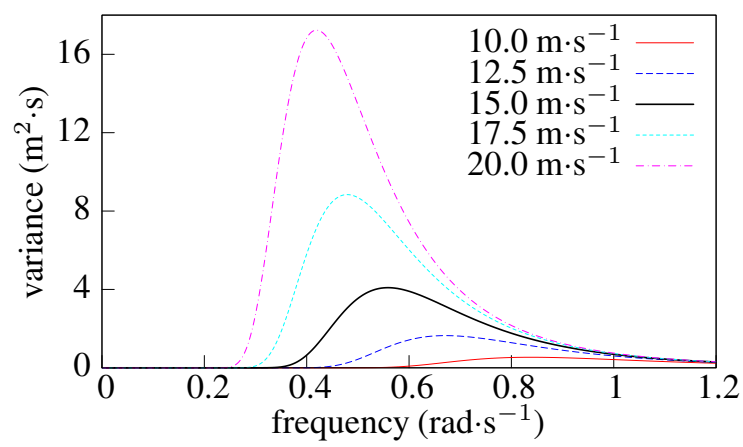

Figure 1: Pierson-Moskowitz frequency spectrum $S_{P M}(\omega)$ with different wind speed values $U_{10}$.

For a fetch limited sea, where waves continue to grow, the JONSWAP spectrum has been found by (Hasselmann and al., 1973) to be more appropriate (figure 2). This is the previous spectrum with an additional term:

$$
S_{J}(\omega)=S_{P M}(\omega) \gamma^{r}
$$

with

$$
r=\exp \left(-\frac{\left(\omega-\omega_{p}\right)^{2}}{2 \sigma^{2} \omega_{p}^{2}}\right) .
$$

Here, $\gamma$ is a peak enhancement factor, i.e. when same values are used for $\alpha$ and $\omega_{p}$ in both spectra, we get $\gamma=S_{J}\left(\omega_{p}\right) / S_{P M}\left(\omega_{p}\right)$. The parameter $\sigma$ controls the width of the peak. Usual parameter values for this spectrum are

$$
\alpha=0.076\left(\frac{U_{10}^{2}}{F g}\right)^{0.22},
$$


where $F$ is the fetch in meters,

$$
\omega_{p}=22\left(\frac{g^{2}}{U_{10} F}\right)^{1 / 3},
$$

$\gamma=3.3$, but can vary between 1 and 7 , and

$$
\sigma= \begin{cases}0.07 & \text { if } \omega \leq \omega_{p} \\ 0.09 & \text { if } \omega>\omega_{p}\end{cases}
$$

As the fetch is an important element of the sea state description, we use the JONSWAP spectrum, rather than the Pierson-Moskowitz one.

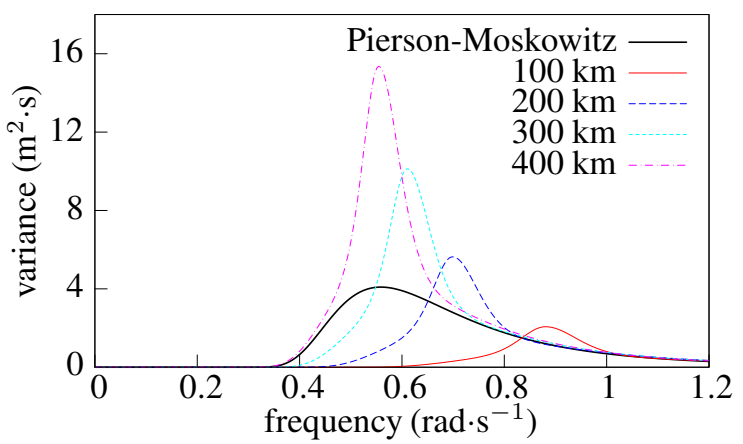

Figure 2: JONSWAP frequency spectrum, $S_{J}(\omega)$, with a wind speed of $U_{10}=15 \mathrm{~m} \cdot \mathrm{s}^{-1}$, and different fetch values $F$.

Other parametric spectrum models can be found in oceanographic literature, including double peaked spectrum for swell coming from distant storm. These spectra are often expressed as a function of frequency $f=\omega /(2 \pi)$. To preserve integral equality, conversion between spectra is done using substitution:

$$
\begin{aligned}
\int S_{f}(f) \mathrm{d} f & =\int S_{\omega}(\omega) \mathrm{d} \omega=\int S_{\omega}(\omega(f)) \frac{\mathrm{d} \omega}{\mathrm{d} f} \mathrm{~d} f \\
& =\int S_{\omega}(2 \pi f) 2 \pi \mathrm{d} f
\end{aligned}
$$

For the dispersion relation, we use the expression found by (Longuet-Higgins, 1962):

$$
D(\omega, \theta)=N(s(\omega)) \cos \left(\frac{\theta_{w}-\theta}{2}\right)^{2 s(\omega)},
$$

where $\theta_{w}$ is the main direction of the spectrum, usually the direction of the wind, and $N(s(\omega))$ is a normalization factor defined as

$$
N(s(\omega))=\frac{1}{2 \sqrt{\pi}} \frac{\Gamma(s(\omega)+1)}{\Gamma(s(\omega)+1 / 2)},
$$

and $\Gamma()$ is the gamma function (figure 3$)$. The function $s(\omega)$ controls the sharpness of the directional spreading and has been defined by (Mitsuyasu and al., 1975) as

$$
s(\omega)=11.5\left(\frac{g}{\omega_{p} U_{10}}\right)^{2.5}\left(\frac{\omega}{\omega_{p}}\right)^{\mu},
$$

with

$$
\mu= \begin{cases}5 & \text { if } \omega \leq \omega_{p} \\ -2.5 & \text { if } \omega>\omega_{p}\end{cases}
$$

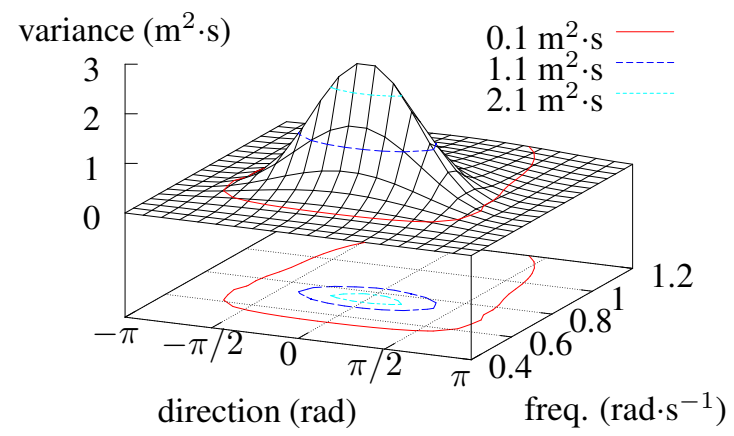

Figure 3: 3D plot of the JONSWAP spectrum, $E_{J}(\omega, \theta)$, with $U_{10}=25 \mathrm{~m} \cdot \mathrm{s}^{-1}, F=100 \mathrm{~km}$ and $\theta_{w}=0 \mathrm{rad}$.

For a simple use, only wind speed, direction and fetch need to be given to the spectrum. In order to make the spectrum curve fit particular data, like oceanic measurements, the default parameter values can be changed, until the desired curve shape is reached (figure 4).

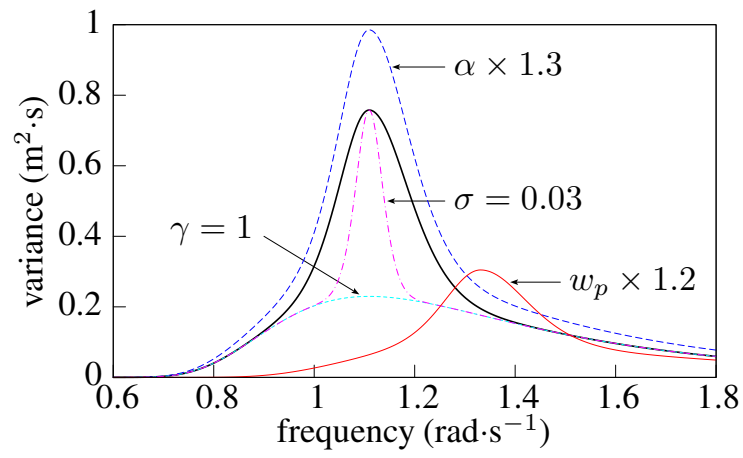

Figure 4: JONSWAP frequency spectrum, $S_{J}(\omega)$, showing how different parameter values alter the curve shape $\left(U_{10}=\right.$ $\left.15 \mathrm{~m} \cdot \mathrm{s}^{-1}, F=50 \mathrm{~km}\right)$.

\subsection{Spectrum sampling}

We now look for a selection of wave frequencies and directions. For the FFT based model, the size and sample number of the rendered surface determine the set of wave vectors to be used (see equation 8). For the parametric one, as there is not such restriction, we select waves that are the most representative of the spectrum energy dispersion. For this purpose, we sample the wave spectrum over a limited domain $\left[\omega_{\min }, \omega_{\max }\right] \times\left[\theta_{\min }, \theta_{\max }\right]$. We want this domain to be the most representative of the whole spectrum, 
i.e. we want it to be as dense as possible. So, by iteration, we found one of the smallest domain that contains roughly $95 \%$ of the total energy.

For simplicity, a regular sampling could be used by taking a constant size $\Delta \omega \times \Delta \theta$ for each sample, with $\Delta \omega=\left(\omega_{\max }-\omega_{\min }\right) / N$ and $\Delta \theta=$ $\left(\theta_{\max }-\theta_{\min }\right) / M$, where $N$ and $M$ are the number of frequency and angle samples, respectively. But an adaptive sampling is more appropriate, especially when few waves are summed, as this better reflects the spectrum attributes. For this purpose, we over-sample parts of the spectrum representing large amount of energy, like (Thon and Ghazanfarpour, 2002). For a given spectrum, we build a quadtree of $4+6 n$ samples, where $n$ is an integer. Samples have different sizes but each of them roughly corresponds to the same energy, and so the same amplitude. Sample centers give us corresponding wave vector polar coordinates (figure 5). So, we now have a set of wave amplitudes, frequencies and directions $(a, \omega, \theta)$. In order to use them with ocean models, we convert frequencies to wavenumbers and polar coordinates to Cartesian ones, to get a set of wave vectors $(\vec{\kappa})$.

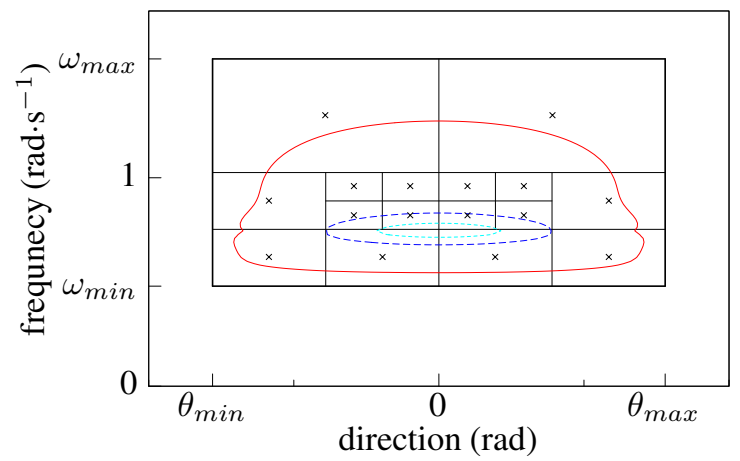

Figure 5: 2D projection of the spectrum from figure 3, showing adaptive sampling with 16 waves.

Using this method, we make oceanic surfaces with realistic wave shapes and global structure which corresponds to the wanted weather condition. However, if a close look at the surface is needed, we have to pay special attention to short waves. When wind speed or fetch increase, energy is transferred to lower frequency waves, i.e. to high wavelength waves (see figures 1 and 2). As we use adaptive sampling, we take more samples for low frequencies and less for high ones, resulting in a smoother surface at a human scale and a lack of realism. So, the spectrum needs to be sampled over a second domain corresponding to short waves, up to the capillary wave size. This domain must be close to the first one, to avoid a "hole" in the whole frequency range. The number of samples we take can vary, depending of the distance between the observer and the surface, in the spirit of (Hinsinger et al., 2002).
For the FFT based model, we sample a spectrum $E(\vec{\kappa})$ in the Cartesian wavenumber domain (figure 6). As the parametric spectra do not give same values for a wave and its opposite (i.e. $E(\vec{\kappa}) \neq E(-\vec{\kappa})$ ), the real number method (equation 9) cannot be used. To preserve integral equality, spectrum conversion is done according to substitution rule. First, we convert the frequency spectrum $S_{\omega}(\omega)$ to a wavenumber spec$\operatorname{trum} S_{\kappa}(\kappa)$ :

$$
\begin{aligned}
\int S_{\kappa}(\kappa) \mathrm{d} \kappa & =\int S_{\omega}(\omega) \mathrm{d} \omega=\int S_{\omega}(\omega(\kappa)) \frac{\mathrm{d} \omega}{\mathrm{d} \kappa} \mathrm{d} \kappa \\
& =\int S_{\omega}(\sqrt{g \kappa}) \frac{1}{2} \sqrt{\frac{g}{\kappa}} \mathrm{d} \kappa
\end{aligned}
$$

Second, we convert the wavenumber directional spectrum $E_{\kappa, \theta}(\kappa, \theta)=S_{\kappa}(\kappa) D(\omega=\sqrt{g \kappa}, \theta)$ from polar coordinates to Cartesian ones:

$$
\int E_{\vec{\kappa}}(\vec{\kappa}) \mathrm{d} \vec{\kappa}=\iint E_{\kappa, \theta}(\kappa, \theta) \frac{1}{\kappa} \mathrm{d} \theta \mathrm{d} \kappa .
$$

Finally, we get

$$
E_{\vec{\kappa}}(\vec{\kappa})=E_{\omega, \theta}(\sqrt{g \kappa}, \theta) \frac{1}{2 \kappa} \sqrt{\frac{g}{\kappa}} .
$$

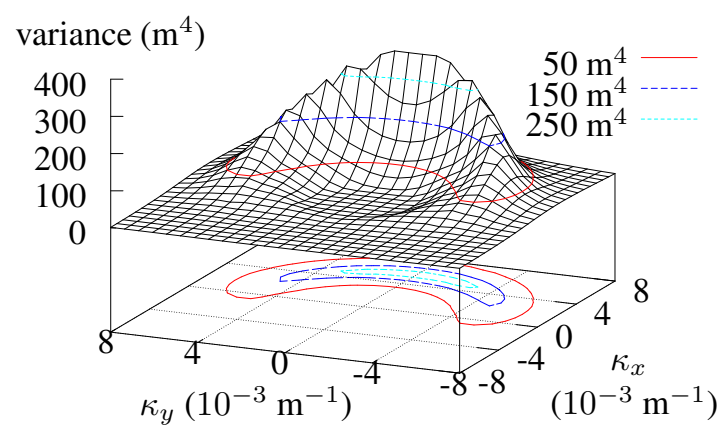

Figure 6: The same spectrum as in figure 3, but in the wave vector domain $\kappa_{x} \times \kappa_{y}$ (i.e. $E_{J}(\vec{\kappa})$ ).

As the wavenumber vectors are set by the rendered surface attributes, we cannot use adaptive sampling. Furthermore, many vectors correspond to a negligible energy amount but are still used. This is somehow balanced by the effectiveness of the FFT algorithm: for the same computation time, we can use a lot more waves, and so spectrum samples, than with the parametric method. However, we have to deal with surface size and sampling on one hand, and with spectrum sampling on the other hand. Looking at equation 8 , we see that absolute values of wave vector components $\kappa_{x}$ and $\kappa_{y}$ are bounded, i.e. $2 \pi / L_{x} \leq$ $\left|\kappa_{x}\right|<\pi N / L_{x}$ and $2 \pi / L_{y} \leq\left|\kappa_{y}\right|<\pi M / L_{y}$. So, we first have to consider surface size $L_{x} \times L_{y}$, as it determines the lowest wavenumber value. Then, 
we choose the number of surface (and so spectrum) samples, knowing that it is related to the highest wavenumber value.

\section{STATISTICAL PROPERTIES OF THE OCEAN SURFACE}

Measurements of oceanic wave statistics have shown that, to a reasonable accuracy, the surface elevation $\eta$ follows a normal distribution. Attributes of oceanic waves we simulate are consistent with these observations. If we look at the oceanic surface as an infinite sum of waves with infinitely small amplitude, we see that the wave spectrum is a probability density function of wave frequencies and directions. Furthermore, the variance of the surface elevation is finite (equation 12). So, by virtue of the central limit theorem, we know that the more waves we sum, the closer to the normal distribution is the simulated surface elevation.

Another observed characteristic of oceanic waves is that their heights follow a Rayleigh distribution, as noted by (Fournier and Reeves, 1986). This distribution is used with a parameter $\sigma=H_{s} / 2$, where $H_{s} \approx$ $4 \sqrt{\operatorname{var}(\eta)}$ is known as the significant height. From this, it can be shown that the probability that a wave has a larger height than $H_{s}$ is $\exp (-2) \approx 0.1353$.

Since they use defined amplitudes, the ocean models presented in section 2 are known as deterministic methods. (Tucker et al., 1984) showed that uniformly distributed random phase terms $\phi$ should be replaced with random amplitudes. An expression like $a \cos \left(\kappa x_{0}-\omega t+\phi\right)$ becomes

$$
\begin{aligned}
& r_{1} a \cos \left(\kappa x_{0}-\omega t\right)+r_{2} a \sin \left(\kappa x_{0}-\omega t\right) \\
& =R a \sin \left(\kappa x_{0}-\omega t+\Phi\right),
\end{aligned}
$$

where $r_{1}$ and $r_{2}$ are random numbers from the standard normal distribution, i.e. with a mean of zero and a standard deviation of one, $R=\sqrt{r_{1}^{2}+r_{2}^{2}}$ follows a Rayleigh distribution and

$$
\Phi= \begin{cases}\arctan \left(\frac{r_{1}}{r_{2}}\right) & \text { if } r_{2} \geq 0 \\ \arctan \left(\frac{r_{1}}{r_{2}}\right)+\pi & \text { if } r_{2}<0 .\end{cases}
$$

This non-deterministic method has better statistical properties than the previous one. In order to use it with parametric and FFT methods, we rewrite equations 4 and 6 according to equation 28 .

\section{RESULTS AND DISCUSSION}

We made a simple real-time implementation of ocean models. We focused on wave shapes and animations, without considering effects like Fresnel reflectivity and transmissivity, or foam and spray (figure 7). Of course, rendering could have been achieved with classical high quality techniques as well. Interactions of objects with ocean surfaces are not specially handled by the Lagrangian model we use, and are beyond the scope of this paper.

Since our method preserves the main spectrum energy, the global structure of the rendered surface is independent of the number of waves we sum. For both ocean models, the sea state we get is always consistent with the provided wind parameters, which cannot be achieved with other existing methods. Since these parameters are the only ones needed to have full control of the method, there is no need for the user to adjust the resulting surface by trial and error.

As previously noted, the FFT model can be particularly tedious to use. To catch a reasonable part of the spectrum, the grid length and the number of samples have to be carefully chosen, whatever are the desired surface characteristics. For example, taking 512 samples up to a frequency of $25 \mathrm{rad} \cdot \mathrm{s}^{-1}$ requires a grid length of no more than $25 \mathrm{~m}$. Furthermore, as this leads to a lowest frequency of about $1.5 \mathrm{rad} \cdot \mathrm{s}^{-1}$, the spectrum peak may be under-sample.

We have tested our implementation with a $3 \mathrm{GHz}$ Pentium 4 PC and a Radeon 9200 graphic board. With the FFT model, we got about $65 \mathrm{fps}$ and $13 \mathrm{fps}$ with, respectively, a grid of $128 \times 128$ and $256 \times 256$ samples. With the parametric equations, we got $8 \mathrm{fps}$ with a regular grid of $128 \times 128$ samples and 50 waves. And taking less than 100 waves leads to poor detailed results. Clearly, only the FFT can reach interactive rate. Although we do not implement an adaptive surface mesh, it seems obvious this could not compete with the FFT speed. Parametric equations should be kept for non-interactive rendering, since they are easiest to use.

\section{CONCLUSION}

We have presented a method for accurate wave energy spectrum sampling that allows realistic ocean surface synthesis and animation. For given wind parameters, the wave heights and directions are computed such that statistical properties of the resulting surface are correct. Since it does not rely on any ocean model, this method is suitable for Gerstner equations and Fourier transforms.

\section{Acknowledgments}

The author wish to thank Bertrand le Saëc and JeanChristophe Gonzato for rereading this paper. 

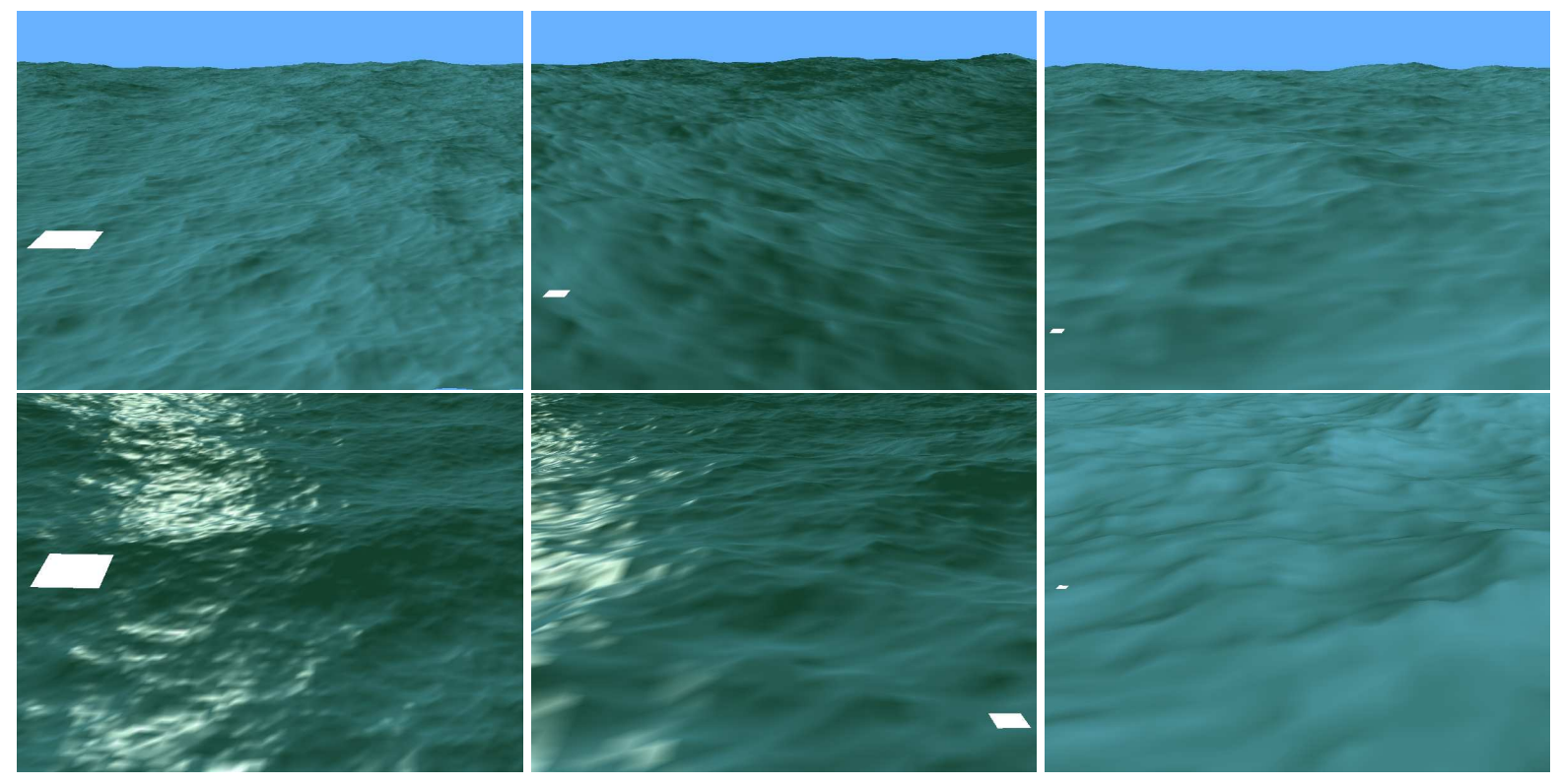

Figure 7: FFT with a grid of $512 \times 512$ samples. Left: grid length is $50 \mathrm{~m}$ and wind speed is $5 \mathrm{~m} \cdot \mathrm{s}^{-1}$. Middle: grid length is $450 \mathrm{~m}$ and wind speed is $15 \mathrm{~m} \cdot \mathrm{s}^{-1}$. Right: grid length is $1500 \mathrm{~m}$ and wind speed is $25 \mathrm{~m} \cdot \mathrm{s}^{-1}$. White square is $1 \mathrm{~m}$ wide.

\section{REFERENCES}

Airy, G. B. (1845). Tides and waves. In Encyclopaedia Metropolitana, volume 5, chapter 192, pages 241-396.

Cieutat, J.-M., Gonzato, J.-C., and Guitton, P. (2001). A new efficient wave model for maritime training simulator. In Spring Conference on Computer Graphics.

Enright, D., Marschner, S., and Fedkiw, R. (2002). Animation and rendering of complex water surfaces. In Conf. on C. G. and interactive techniques, pages 736-744.

Fournier, A. and Reeves, W. T. (1986). A simple model of ocean waves. SIGGRAPH Computer Graphics, 20(4):75-84.

Gamito, M. N. and Musgrave, F. K. (2002). An accurate model of wave refraction over shallow water. Computers \& Graphics, 26(2):291-307.

Gonzato, J.-C. and le Saëc, B. (2000). On modeling and rendering ocean scenes. Journal of visualisation and computer simulation, 11:27-37.

Hasselmann, K. and al. (1973). Measurements of windwave growth and swell decay. Ergänzungsheft zur Deutschen Hydrographischen Zeitschrift, (12).

Hinsinger, D., Neyret, F., and Cani, M.-P. (2002). Interactive animation of ocean waves. In Symposium on Computer Animation, pages 161-166.

Iglesias, A. (2004). Computer graphics for water modeling and rendering: a survey. Future generation computer systems, 20(8):1355-1374.

Longuet-Higgins, M. S. (1962). The distribution of intervals between zeros of a stationary random function. Philosophical Transactions for the Royal Society of London, 254:557-599.
Mastin, G. A., Watterberg, P. A., and Mareda, J. F. (1987). Fourier synthesis of ocean scenes. IEEE Computer Graphics and Applications, 7(3):16 - 23.

Mihalef, V., Metaxas, D., and Sussman, M. (2004). Animation and control of breaking waves. In Symposium on Computer Animation, pages 315-324.

Mitsuyasu, H. and al. (1975). Observations of the directional spectrum of ocean waves using a cloverleaf buoy. Jour. of physical oceanography, 5(4):750-760.

Peachey, D. R. (1986). Modeling waves and surf. SIGGRAPH Computer Graphics, 20(4):65-74.

Phillips, O. M. (1957). On the generation of waves by turbulent wind. Journal of fluid mechanics, 2:417-445.

Pierson, W. J. and Moskowitz, L. (1964). A proposed spectral form for fully developed wind seas. Journal of geophysical research, 69:5181-5203.

Premože, S. and Ashikhmin, M. (2001). Rendering natural waters. Computer Graphics Forum, 20(4):189-199.

Rankine, W. J. M. (1863). On the exact form of waves near the surface of deep water. Philosophical transactions of the Royal society of London, pages 127-138.

Tessendorf, J. (2001). Simulating ocean water. ACM SIGGRAPH course notes. Updated in 2004.

Thon, S. and Ghazanfarpour, D. (2002). Ocean waves synthesis and animation using real world information. Computers and Graphics, 26(1):99-108.

Tucker, M. J., Challenor, P. G., and Carter, D. J. T. (1984). Numerical simulation of a random sea. Applied ocean research, 6(2):118-122.

von Gerstner, F. J. (1804). Theorie der wellen. Abhandlungen der Königlichen Böhmischen Gesellschaft der Wissenschaften, 1:1-65. 